\title{
CONSTRUCCIÓNY VALIDACIÓN DE LA ESCALA DE AUTOEFICACIA PARA EL USO DEL CONDÓN EN ADOLESCENTES MEXICANOS
}

CONSTRUCTION AND

VALIDATION OF THE

SELF-EFFICACY SCALE TO THE USE OF CONDOM IN MEXICAN ADOLESCENTS

Recibido enero 2016 Aceptado abril 2016

Correspondencia: Landeros Olvera Erick E_mail: erick_landeross@hotmail.com nursingresearch@outlook.com

\section{Autores:}

Carrera Huerta Saudi

Pasantes de la Licenciatura en Enfermería Benemérita Universidad Autónoma de Puebla (BUAP)

Soto Carrasco Ana Amelia

Pasantes de la Licenciatura en Enfermería Benemérita Universidad Autónoma de Puebla (BUAP)

Muñoz Muñoz Vivian Paola

Pasantes de la Licenciatura en Enfermería Benemérita Universidad Autónoma de Puebla (BUAP)

Sánchez Salinas Reina

Pasantes de la Licenciatura en Enfermería Benemérita Universidad Autónoma de Puebla (BUAP)

Osorio Ramírez Jenny Lizbet

Pasantes de la Licenciatura en Enfermería Benemérita Universidad Autónoma de Puebla (BUAP)

Abeille Mora Erika

Pasantes de la Licenciatura en Enfermería Benemérita Universidad Autónoma de Puebla (BUAP)

Landeros Olvera Erick

Doctor en Ciencias de Enfermería. Docente-Investigador de la BUAP. Miembro del Sistema Nacional de Investigadores (BUAP)

Palabras clave: Adolescentes, autoelicacia, condón

Key words: Adolescents, self-eflicacy, condom 


\section{RESUMEN}

Introducción: Entre los adolescentes, las infecciones de transmisión sexual y embarazos, pueden ser combatidos a trovés del uso del condón. Es importante no solo educar, si no determinar la habilidad para su uso. Es aquí donde cobra importancia la autoeficacia, elemento cognitivo que determina la confianza que el adolescente tiene para usar correctamente el condón. En México y Latinoamérica, no se ha evidenciado la construcción y validación de un instrumento exclusivo para el uso del condón en adolescentes en el idioma español y que sea culturalmente factible.

Objetivo: Construir y validar un instrumento que evalúe la autoeficacia del uso del condón en adolescentes,

Metodología: Diseño de proceso de alcance transversal. La muestra estuvo conformada por 130 adolescentes.

Resultados: El análisis estadístico incluyó la técnica de jueces con un Índice de Validez de Contenido de 8.64. Alfa de Cronbach de .086, KMO=.795, Esfericidad de Bartlett p<.05. En análisis factorial fue exploratorio, los primeros 5 ítems explicaron más del 70\% de la varianza y al rotar los factores se evidenciaron 4 grupos. La versión final del instrumento consta de 17 ítems, con un formato de respuesta tipo Likert representado por caritas, que corresponden a 7=Nunca, hasta $5=$ Siempre.

Conclusión: Se obtuvo un instrumento creado en el contexto cultural del territorio mexicano que mide la Autoeficacia del Uso del Condón en los Adolescentes, sin embargo, se requiere poner a prueba el instrumento en otros contextos culturales del territorio mexicano para probar su validez externa.

Palabras clave. Adolescentes, autoeficacia, condón.

\begin{abstract}
Introduction: Among adolescents sexual transmitted infections and pregnancies can be combated through the use of condoms. It is important not just educate, but determine the skills for the use of condom. Here is where self-efficacy grows in importance, since it's a cognitive element to determine the confidence the adolescent has to use the condom correctly. In Mexico and Latin-Amerioa there is no evidence on the construction and validation of an instrument exclusively on the use of condoms in adolescents in Spanish and culturally feasible.
\end{abstract}

Objective: Construct and validate an instrument to evaluate the adolescents' self-efficacy in the use of condom.

Method: Design in process to transversal scope. One hundred thirty adolescents were included in the sample.

Results: The statistical analysis included the judges' technique with a content validity index of 8.64. Cronbach Alpha .86, $\mathrm{KMO}=.795$, Bartlett Sphericity $\mathrm{p}<$.05. The factor analysis was exploratory, and the first 5 items explained more than 70\% of variance and when factors rotate 4 groups were evident. The instrument final version consists of 17 items with a Likert-type response format represented by small faces and corresponding to $7=$ never, to $5=$ always.

Conclusion: An instrument created within the Mexican territory cultural context was obtained. It measures the adolescents' self-efficacy to the use of condom; however, it is necessary to test the instrument in other cultural context different from the Mexican context to prove its external validity.

Key words. Adolescents, self-efficacy, condom. 


\section{INTRODUCCIÓN}

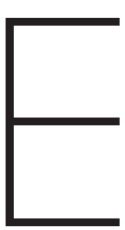

I mayor reto de salud en los adolescentes en este siglo responde a tres problemáticas específicas: VH/SIDA: infecciones de transmisión sexual (ITS) y embarazos no deseados que conllevan a abortos y muertes perinatales. Estos problemas en conjunto representan un reto de sanidad pública.

A nivel mundial, cada día 7,100 personas contraen VIH/SIDAy aproximadamente 34 millones anualmente. América Latina se encuentra en tercer lugar con alrededor de 1.5 millones de personas con VIH. De los cuales 68000 son adultos jóvenes; siendo 34 680 mujeres y el resto son hombres; quienes durante su adolescencia comenzaron su vida sexual activa y adquirieron el virus.?

En México durante el año 2010 se estimó la ocurrencia de 10,000 nuevas infecciones por $\mathrm{VIH}$, el 50\% se produjeron en personas entre los 10 y 24 años de edad, siendo la sexual la vía de transmisión más importante en $96.1 \%$ en hombres y 93.5\% en mujeres. ${ }^{2}$

De acuerdo con la última Encuesta Nacional de Salud y Nutrición (ENSANUT) 3.7.1\% de los adolescentes tuvieron su primera relación sexual antes de los 15 años y con base al registro nacional de casos de SIDA de un total de 153, 109, el 82\% son hombres y el 18\% mujeres.

En el mismo orden de ideas, las ITS representan un problema serio de salud pública en adolescentes mexicanos; el periodo del 2005 al 2010 la candidiasis urogenital y el VPH han sido las infecciones de mayor incidencia en jóvenes de 15 a 24 años de edad con un total de 785.25 por cada 100,000 habitantes a nivel nacional. ${ }^{4}$ Según cifras reportadas por la ENSANUT,3 se encontró una proporción mayor del porcentaje de adolescentes de 12 a 19 años de edad que han iniciado vida sexual, dado que la cifra actual alcanza 23\%, cuando en 2006 se reportó para esto mismo 15\%, de los cuales el 2.3\% reportó actividad sexual protegida. Estos datos permiten ver una proporción importante de adolecentes mexicanos que están llevando a cabo prácticas sexuales no protegidas que ponen en riesgo su salud. Cabe mencionar que en la adolescencia se despierta el interés por el cuerpo y la sexualidad consolidándose las actitudes y comportamientos que incidirán en la adopción o no de prácticas sexuales más seguras. ${ }^{5}$

En lo que respecta al embarazo no deseado, abortos y muerte perinatal, representa un reto constante en los países en vías de desarrollo por el impacto a los indicadores de salud y sociales. El mayor riesgo de mortalidad materna corresponde a las adolescentes de menos de 15 años. Las complicaciones del embarazo y el parto son la principal causa de muerte de las adolescentes en la mayoría de los países en desarrollo.

Cabe mencionar que los embarazos no deseados es otro de los problemas por practicar relaciones sexuales sin protección, de ahí el hecho que la tasa de fecundidad aumentó de 30 a 37 embarazos por cada 1000 mujeres de 12 a 19 años que iniciaron vida sexual activa, de lo que va del 2005 al 2012 y como consecuencia también conlleva abortos clandestinos y muertes perinatales ${ }^{3}$. Con respecto a los abortos, Juárez et al. ${ }^{6}$, mencionan que en México ocurren aproximadamente un millón de abortos al año. La tasa anual de abortos inducidos en nuestro país es de 38 abortos por cada mil mujeres siendo el grupo de edad prevalente, adolescentes de 15 a 19 años. Sin embargo no se encuentran datos que indiquen el número de abortos practicados (clandestinos) en adolescentes actualizado, podemos suponer que aunque no se tenga el dato, representa un problema grave de salud pública a pesar de que en la Capital de México ésta práctica sea legal. Con respecto a las defunciones perinatales durante el año 2012 fueron de 18,151 muertes. Datos que representan 63\% del total, considerando que uno de los primeros factores de riesgo para ello, son las adolescentes embarazadas ${ }^{3}$

Los tres problemas en cuestión pueden ser combatidos con educación para la salud sexual, fortaleciendo los

\footnotetext{
72 nim $/$ Why Enf Neurol Vol. 15. No. 2 mayo - agosto 2016
} 
valores y la convicción de la abstinencia sexual en la adolescencia. Sin embargo, las prácticas sexuales en adolescentes es una realidad que el sistema de salud debe reconocer y atender. Es aquí donde es importante desarrollar y aplicar programas de promoción y prevención en salud sexual. Uno de los métodos más seguros es a través del uso del condón que representa prácticas sexuales que disminuyen los riesgos. A pesar de que se conoce y se difunde el uso del condón, las estrategias no han sido efectivas dado los indicadores epidemiológicos previamente mencionados. Se requiere fortalecer esta práctica a través de teoría cognitiva e indicadores empíricos que muestren el grado de conocimiento o seguridad de utilizar correctamente un condón.

Una de las teorías que mejor resultado han tenido es la Cognitiva social de Albert Bandura (1986) ${ }^{7}$ que utiliza la autoeficacia (AE) para conocer las creencias de cada individuo en realizar conductas de forma exitosa. La AE pude ser especializada o direccionada; por ejemplo, para el uso del condón AE se define como la confianza en sus capacidades para utilizar el condón de forma correcta en cada relación sexual. Teóricamente, esto predice la acción y constituye una herramienta de protección, dado que existe una correlación alta en lo que un individuo cree que puede realizar con lo que realmente ejecuta. En otras palabras, a mayor AE, mayor protección.

Diversos instrumentos han sido construidos para medir la autoeficacia (AE) en el uso del condón de manera general. Los cuales han sido aplicados en población de adolescentes ${ }^{5,8}$ y adultos jóvenes principalmente 8,9,10,17,12 con validaciones internas y estructura factorial aceptables 5,9,10. Asimismo los tipos de estudios que utilizaron estos instrumentos han sido descriptivos ${ }^{17}$. transversales ${ }^{8,10,12}$ y correlacionales ${ }^{12}$. Se han adaptado al español algunos instrumentos, que entre sus dimensiones se encuentra autoeficacia para el uso del condón en adolescentes ${ }^{5}$, adolescentes y adultos ${ }^{10}$ comportamientos del uso del condón en adultos jóvenes universitarios ? . Sin embargo, en México y Latinoamérica, no se ha evidenciado la construcción y validación de un instrumento exclusivo para el uso del condón en adolescentes en el idioma español y que sea culturalmente factible.

Por lo tanto el objetivo de este estudio es construir y validar un instrumento que evalúe la autoeficacia del uso del condón exclusivamente en adolescentes que pudiera servir en proyectos futuros para fortalecer las intervenciones del sistema de salud.

\section{METODOLOGÍA}

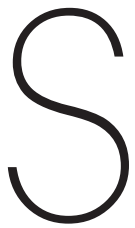

e realizó un diseño de proceso de alcance transversal ${ }^{13}$ para construiry validar un instrumento que mida empíricamente la autoeficacia del uso del condón en adolescentes mexicanos.

La población estuvo conformada por adolesoentes estudiantes del nivel medio superior. El muestreo se realizó por conveniencia en una preparatoria pública, sin embargo la selección de los participantes fue aleatoria al interior de los grupos por medio de las listas de asistencia. El tamaño de la muestra se estimó mediante una potencia de $90 \%$ y nivel de significancia de 0.5, con la fórmula para poblaciones finitas:

$\frac{n=z u 2(p)(q)}{d 2}$

\section{Donde: $\mathrm{n}$ = tamaño de la muestra finita \\ zu2 =1.96 ( $z=$ confiabilidad de 90\%) \\ $\mathrm{p}=$ probabilidad de éxito \\ $q=$ probabilidad de fracaso \\ d2=error máximo permitido $(0.05)$}

Quedando así un resultado con un muestra de 119 participantes. Para amortiguar el efecto de atrición se aumentó un 10\% el tamaño de la muestra quedando una $n=130$ para la muestra final.

Criterios de selección: Se incluyeron adolescentes que se encontraban cursando el último año del nivel medio superior, igual o menor de 19 años de edad, que han iniciado vida sexual y que el padre o tutor haya firmado previamente el consentimiento informado para la aplicación del instrumento. Se 
excluyeron a todos aquellos adolescentes que no se encontraban presentes en el día y momento de la aplicación del instrumento de acuerdo a la lista presentada por el docente. Se eliminaron a los estudiantes que ya no desearon participar.

\section{Procedimientos}

1. Construcción semántica de los reactivos que constituyen el instrumento. Con base al concepto de autoeficacia los reactivos puntualizan solo en el uso del condón, no se enfatizó en otros elementos que no tuvieran relación con tipos de condón, lugares para comprar el condón, número de parejas sexuales, entre otras.

2. Validación del instrumento por técnica de jueces. Los jueces fueron invitados de un programa doctoral, tanto a Doctorados en Ciencias de Enfermería y estudiantes de cuarto año. El análisis estadístico de las respuestas de los jueces se llevó de acuerdo a Kaltz y colaboradores. ${ }^{14}$ Los resultados de ésta validación proporcionaron las herramientas necesarias para la construcción de la segunda versión del instrumento de acuerdo a las observaciones y cambios sugeridos por los jueces.

3. Prueba piloto. El instrumento fue aplicado a una muestra de 30 adolescentes estudiantes del último año del nivel medio superior de una escuela pública de la ciudad de Puebla, México. El objetivo es que con los resultados de la prueba piloto se construya la tercera versión del instrumento.

4. Aplicación de la versión final del instrumento y análisis estadístico del instrumento. Previo consentimiento informado, el instrumento fue sometido a un análisis de confiabilidad de alfa de Cronbach y análisis factorial por técnica Varimax. Para el tratamiento de los datos se utilizó el programa estadístico SPSS versión 21.

Consideraciones Éticas

La recolección de los datos se llevó a cabo de acuerdo con las disposiciones de la Ley General de
Salud en Materia de Investigación ${ }^{15}$. Titulo Quinto "investigación para la salud" del Capitulo Único. El estudio se considera de riesgo mínimo dado que no se manipuló física ni emocionalmente a los participantes. Del mismo modo se les proporcionó un consentimiento informado una semana antes de contestar el instrumento, el cual fue firmado por los que tenían mayoría de edad y por su padre o tutor en aquellos menores de edad considerando el asentimiento de los menores. Durante la obtención de los datos se respetó la confiabilidad de la información de cada participante, para cumplir con lo estipulado en el Titulo Quinto Capítulo Único Fracción IV. En el mismo instrumento se preguntó si habían iniciado vida sexual, las respuestas negativas, fueron instrumentos que se excluyeron y no fueron evidenciados ante el grupo de adolescentes, conservando la confidencialidad. De igual manera los datos fueron manejados únicamente por el grupo de investigadores, dichos instrumento fueron resguardados en un sobre después de ser contestados por los participantes.

\section{Resultados}

1. Bajo una revisión y análisis del concepto de autoeficacia, los investigadores construyeron 14 ítems, cuya redacción semántica enfatizaba únicamente en la autoeficacia para el uso del condón. La escala cuenta con un formato tipo Likert con cinco opciones de respuesta que van desde 1) Nunca, 2) Casi nunca, 3) Algunas veces, 4) Casi siempre, hasta 5) Siempre. El valor mínimo es de 14 puntos y el máximo es de 70 puntos. No se establecieron puntos de corte o transformaciones de los datos en índices de cero a cien, se determinó que entre más alto sea el puntaje, más alto es la autoeficacia para en el uso del condón. Así mismo no se establecieron las dimensiones para agrupar a priori los îtems.

\section{4}


2. Una vez construida la primera versión, se realizó una validación por diez jueces cuyos resultados fueron los siguientes:

Tabla 1. Resultados de la Validación de Jueces

Rubro
Resultado

Así mismo los jueces sugirieron modificaciones a la redacción de algunos îtems para conservar la dirección hacia el concepto de autoeficacia.

Con base a las correcciones sugeridas por los jueces, se realizó una segunda versión del instrumento, anexando tres ítems a los ya establecidos.

Los jueces sugirieron agregar una escala análoga visual con imágenes animadas que relacionaban la expresión con la escala tipo Likert originalmente propuesta. El propósito es que el instrumento fuera visualmente más atractivo para los adolescentes para ser contestado.

3. Para la prueba piloto, se aplicó la segunda versión del instrumento a un total de 30 adolescentes en un rango de edad de 16 a 19 años de edad,
Moda igual a 17 años; predominó el género masculino en un 57.7\%.

Con esta prueba se consideraron dos cambios sustanciales: a) cambios en la redacción de algunos îtems del instrumento, remplazando palabras por algunas otras de uso más común entre los adolescentes sugeridas por ellos mismos; b) se eliminó de la escala de caritas, las opciones de frecuencia (entre siempre y nunca). De tal manera que se creó una versión final del instrumento con 17 ítems para la obtención final de los datos.

4. La versión final del instrumento, se aplicó a 130

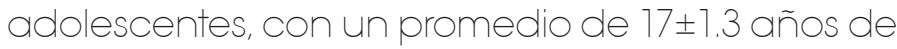
edad, moda igual a 17, predominó el sexo masculino con 52\%. El análisis estadístico inicial se resume en la siguiente tabla.

Tabla 2. Resultados estadístico preliminares.

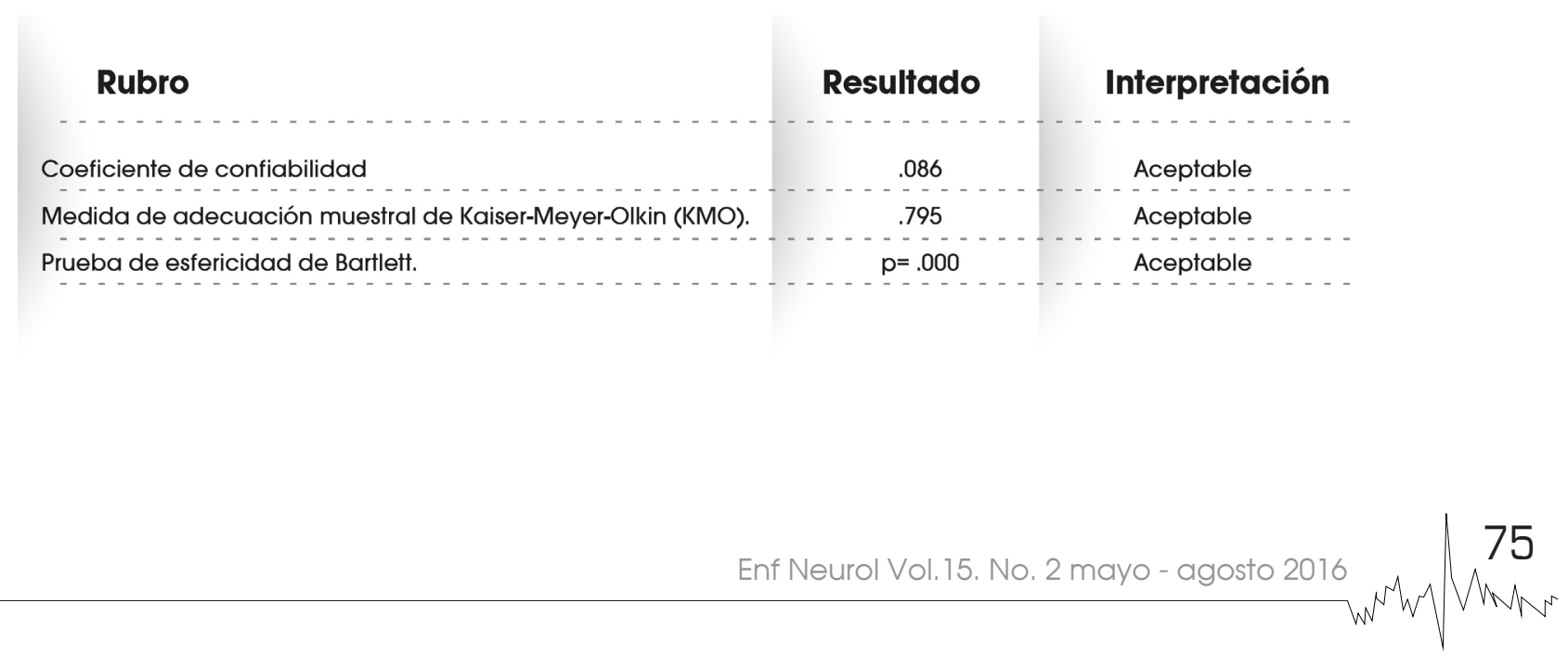


Una vez obtenidos los resultados KMO y la esfericidad de Bartlett, se procedió a realizar una rotación de factores con técnica varimax de forma exploratoria. El propósito fue agrupar los ítems de acuerdo al porcentaje de la variación explicada de cada uno de los îtems, se observó que los primeros 5 ítems explican más del 70\% de la varianza, como se muestra en la siguiente tabla:

Tabla 3. Porcentaje de la varianza explicada por ítem

\begin{tabular}{|c|c|c|c|}
\hline \multirow[b]{2}{*}{ Número de ítem } & \multicolumn{3}{|c|}{ Autovalores iniciales } \\
\hline & Total & $\%$ de la varianza & $\%$ acumulado \\
\hline 1 & 6.069 & 35.698 & 35.698 \\
\hline 2 & 1.965 & 11.562 & 47.259 \\
\hline 3 & 1.644 & 9.672 & 56.932 \\
\hline 4 & 1.151 & 6.770 & 63.702 \\
\hline 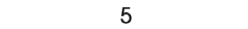 & 1.117 & 6.573 & 70.275 \\
\hline
\end{tabular}

Para complementar la tabla anterior se muestra el gráfico de sedimentación

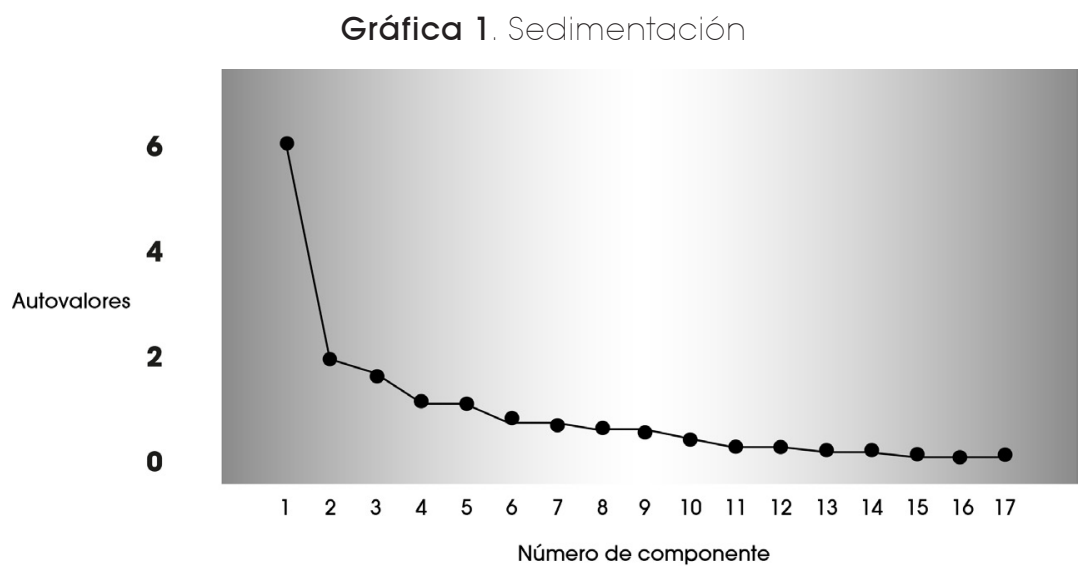

Al realizar la rotación de factores se pudo identificar a los ítems de agrupamiento que pudieran sugerir subescalas al instrumento. El resultado presentó cuatro agrupaciones como se muestra en la siguiente tabla.

Tabla 4. Agrupación de ítems después de la rotación varimax

\begin{tabular}{|c|c|}
\hline & Número de reactivos \\
\hline 1 & $1,8,9,13,14,17$ \\
\hline 2 & $2,3,5,6,7,12$ \\
\hline 3 & $4,10,11$ \\
\hline 4 & 15,16 \\
\hline
\end{tabular}




\section{CONCLUSIÓN}

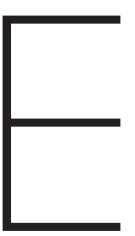

l objetivo de este estudio fue construir y validar un instrumento que evalúe la autoeficacia del uso del condón exclusivamente en adolescentes; dado que no hay instrumentos similares en población latinoamericana, se realiza la discusión puntualizando algunas situaciones del método y del análisis estadístico.

Para la construcción del instrumento, en cada uno de los ítems se enfatizó la "habilidad para hacer - llevar a cabo cierta acción con éxito", se destacó la confianza que el adolescente cree poseer para llevar a cabo el uso correcto del condón. Con esta premisa en la construcción de los ítems, se acercó cada uno de ellos al concepto teórico. Sin embargo después de realizar la validación de jueces y la prueba piloto, en la redacción de los ítems se consideraron cambios cualitativos que mejoraron la validez de constructo.

Con respecto a la validación de jueces, el análisis estadístico resultó ser aceptable y el índice de validez por ítem y de contenido resultaron aceptables, a pesar del 13\% de posibilidad de error de acuerdo al criterio de validez; sin embargo es menor a un 20\% como sería lo permitido en un error tipo Beta (poder estadístico de .80) si el instrumento fuera aplicado a la población adolescente en otros contextos. Estos resultados más la confiabilidad de alfa de Cronbach en la prueba final, denotó una correlación entre los ítems, permitiendo suponer que el instrumento mide lo que dice medir.

Otro de los cambios al instrumento original, consistió en eliminar de la escala de caritas, las opciones de frecuencia (entre siempre y nunca) ya que logramos detectar que formaban parte de las opciones de respuesta y desviaban la atención de las imágenes animadas, las cuales resultaron útiles para identificar la respuesta con su nivel de confianza, dada la expresión de cada carita. Sin embargo en la hoja preliminar al instrumento en donde van las instrucciones, no se excluyeron las opciones de respuesta con relación a las caritas.
Los instrumentos encontrados en la literatura, resultaron ser muy generales con respecto a la medición del concepto y dirigidos a adultos, por ejemplo, "puedo evitar situaciones que puedan llevar al sexo no seguro (tener dos o más parejas sexuales, prostitución, influjo del alcohol u otras drogas", "Puedo hablar con mi pareja sobre usar un condón ante de ponerme muy elevado". A pesar de que se mide la autoeficacia para el uso del condón, por un lado, no está dirigido a la habilidad o técnica para su uso correcto, se hace referencia más a situaciones de riesgo, que son más comunes en adultos, que a las situaciones a las que se enfrenta un adolescente que inicia vida sexual activa. Por otro lado, están escritos en inglés y la traducción no es culturalmente factible para el contexto mexicano. Ante esta situación, para poder llegar al lenguaje de cada adolescente, en nuestro instrumento, algunos ítems cambiaron sus expresiones para poder ser más "amigable" o "comprensible" para el adolescente. Por esta razón es que de la prueba piloto, se consideraron todas las observaciones que los adolescentes comentaron y que en conjunto con las observaciones previas de los jueces, permitieron construir ítems que consideramos adecuados para el adolescente mexicano, por ejemplo: -Aunque sea solo para meter la "puntita", me colocaría el condón ( o se colocaría a mi pareja); -Puedo negarme a tener un "faje" sin usar condónEstas son algunas de las expresiones que no se encontraron en ninguno de los instrumentos validados en otros contextos culturales.

En análisis factorial fue exploratorio, dado que es un instrumento de reciente creación, únicamente se pretendió observar su comportamiento en la variabilidad. El porcentaje de varianza de los primeros cinco ítems fue mayor del esperado en comparación con otros análisis factoriales publicados (más del 70\%), lo que permite confirmar que el concepto está siendo medido. Al realizar la rotación de factores, el modelo mostró cuatro posibles dimensiones, que para esta primera aplicación del instrumento no consideramos asignarles un nombre, dado que requiere de ser aplicado a una muestra mayor para obtener una mejor rotación de factores.

Enf Neurol Vol. 15. No. 2 mayo - agosto 2016 
Como producto final se obtuvo un instrumento creado exclusivamente en el contexto cultural del territorio mexicano que mide la Autoeficacia del Uso del Condón en los Adolescentes, sin embargo, se requiere poner a prueba el instrumento en otros contextos culturales del territorio mexicano para probar su validez externa. Asimismo, obtener una muestra mayor para aumentar la potencia estadística y una variabilidad suficiente para rotar los îtems y asignarles un nombre con el apoyo de la literatura. Así mismo, el instrumento podría someterse a un diseño test, re-test con la finalidad de conocer su consistencia interna bajo un diseño experimental que manipule la autoeficacia de uso correcto del condón a través de una intervención educativa planificada y controlada con grupos paralelos.

Este instrumento puede representar una herramienta única para probar que las intervenciones educativas de enfermería tienen impacto en la confianza de los adolescentes al aumentar su autoeficacia, será un predictor positivo para el uso del condón cuando se inicie una vida sexual e incluso si ya se tiene; así, de forma indirecta, es posible que se impacte en la reducción de infecciones de transmisión sexual y embarazos no deseados.

Conclusión. La construcción del instrumento denominado "Autoeficacia en el Uso del Condón en Adolescentes Mexicanos", resultó ser válida en la muestra seleccionada. De manera preliminar se puede concluir que el instrumento mide el concepto que dice medir y es adecuado para adolescentes y culturalmente específico para el contexto mexicano.

\section{Agradecimientos:}

A los estudiantes del programa de Doctorado en Ciencias de Enfermería de la Universidad de Guanajuato Campus Celaya-Salvatierra. A los estudiantes de las preparatorias BUAP.

\section{REFERENCIAS BIBLIOGRÁFICAS}

1. Organización de las Naciones Unidas sobre SIDA (ONUSIDA) (Internet') 2013. Disponible en: http://mww.unaids.org/es/resources/documents/2013/

2. Centro Nacional para la Prevención y el Control de VIH y el SIDA (CENSIDA) (Internet) 2013. Disponible en: http://www.censida.salud.gob.mx/descargas/ atencion/Censidainforma

3. Secretaría de Salud: Encuesta Nacional de Salud y Nutrición (ENSANut) (Internet) 2012. Disponible en: http://ensanut.insp.mx/informes/ENSANUT2012ResultadosNacionales.pdt

4. Instituto Nacional de Estadística, Geografía e Informática (INEGI) (Internet) 2010. Disponible en: http://www.inegi.org.mx/est/contenidos/proyectos/ccpv/ cpr2010/Default.aspx

5. López, F. Moral, J. Validación de una escala de autoeficacia para la prevención del SIDA en adolescentes. Sal Pub Mex. 2004; 43(5): 421-432

6. Juárez F y Singh S. Incidencia del aborto inducido por edad y estado. México, 2009: nuevas estimaciones usando una metodología modificada. Perspectivas Internacionales en Salud Sexual y Reproductiva. 2009: número especial de 2013: pp. 25-35.

7. Bandura, A., Social Foundations of Thought \& Action. A Social Cognitive Theory. 7th ed. New Jersey; 1986. 390 p.

8. Jiménez, M. Comunicación sexual en adolescentes y su implicación en la consistencia del uso del condón. Enseñanza e Investigación en Psicología México. 2010; 15(1):107-129.

9. Piña, J., Robles, S., Rivera, B. Instrumento para la evaluación de variables psicológicas y comportamientos sexuales de riesgo en jóvenes de dos centros universitarios de México. Rev Pan Sal Pub. 2007: 22(5): 295-303.

10. Hernández, A., López, M., Adaptación Transcultural de la escala para medir autoeficacia en el uso del condón masculino. Index de Enfermería España. 2011; 20 (3):194-198.

11. Uribe, A., Orcasita, L. Evaluación de conocimientos, actitudes, susceptibilidad y autoeficacia frente al $\mathrm{V} / \mathrm{H} /$ sida en profesionales de la salud. Avances en Enfermería. Colombia. 2011; Vol. XXIX (2): 271-284.

12. Ÿlvarez, C., Villarruel, A., Zhou, Y., \& Gallegos, E. Predictors of condom use among mexican adolescents, Research and Theory for Nursing Practice: An International Journal, 2010; 24(3): 187-196

13. Feinstein A. R. Clinical epidemdogy the architecture of clinical research 7th ed. Philadelphia, PA: WB Saunders. 1985

14. Carolyn Feher Waltz, Ora Lea Strickland, Elizabeth R. Lenz. Measurement in Nursing and Health Research. 3th ed. Springer. 2005

15. Ley General de la Salud en Materia de Investigación para la salud 1987 (Internet) 2015. Disponible en http://wnw.salud.gob.mx/unidades/cdi/nom/ compilrlgsmis.html.

\section{8} IMN/Why Enf Neurol Vol.15. No. 2 mayo - agosto 2016 


\section{Instrumento de Autoeficacia en el Uso del Condón en Adolescentes Mexicanos}

(Carrera-Huerta S.; Soto-Carrasco AA; Muñoz-Muñoz VP; Sánchez-Salinas R; Osorio-Ramírez JL; Abeille-Mora E; Landeros-OIvera E.2015)

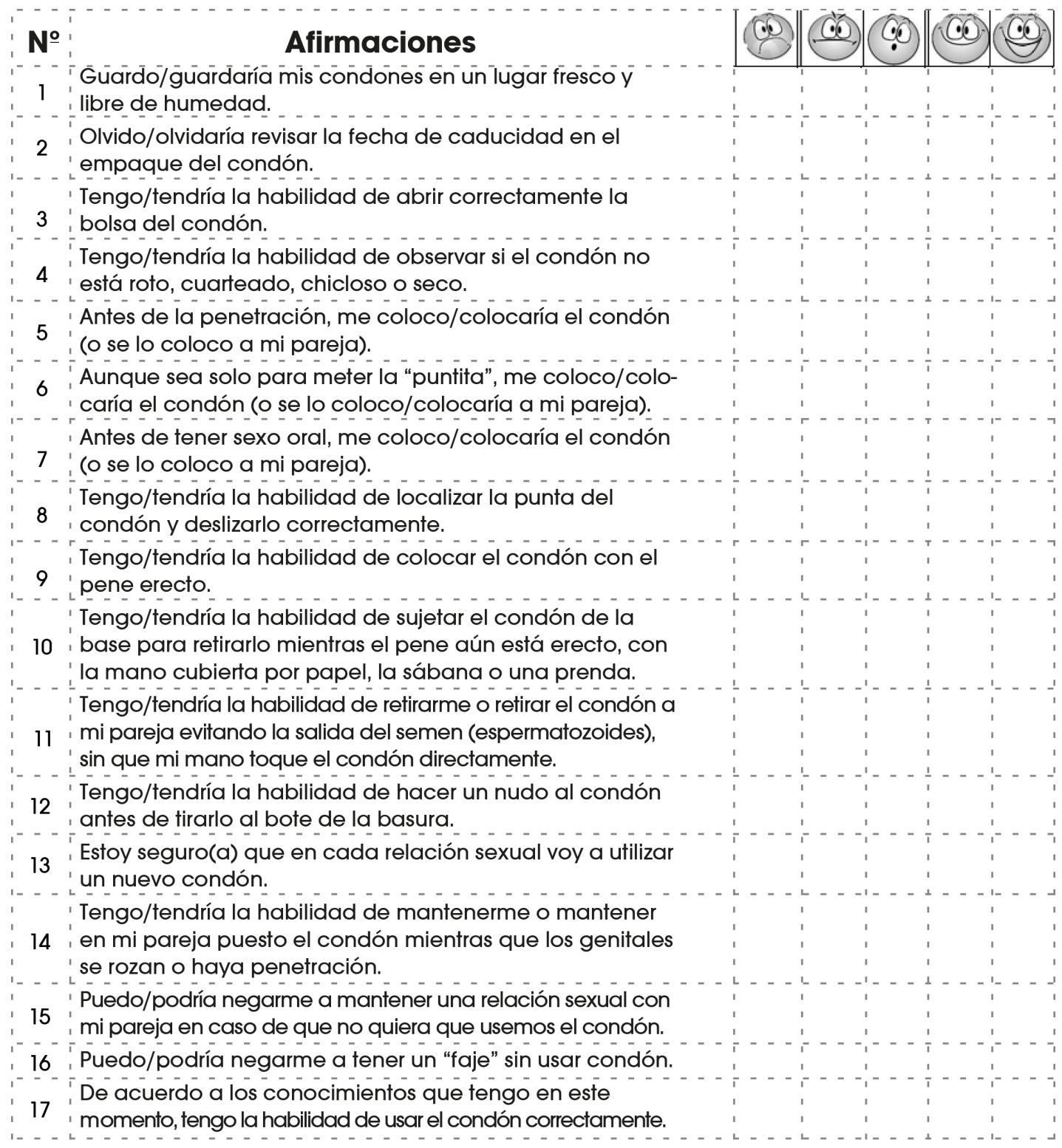

